\title{
Praxistheorie und ethnologische Praxis Überlegungen zu Anwendungsfeldern der Ethnologie
}

\author{
MARTIN SÖKEFELD
}

\begin{abstract}
Die herkömmliche Gegenüberstellung von praktischer/angewandter Ethnologie und wissenschaftlicher/theoretischer Ethnologie verkennt, dass Praxis auch für die „wissenschaftliche“ (universitäre) Ethnologie seit langem eine ganz zentrale Kategorie ist (z. B. Ortner 1984). Universitäre Ethnologie ist keineswegs nur „theoretisch“. Dies gilt in zweifacher Hinsicht: Zum einen ist die universitäre Ethnologie selbst ein Komplex von Praktiken, zum anderen ist Praxis ein unverzichtbares Konzept ethnologischer Analyse. Der Beitrag versucht, die Differenz von universitärer und außeruniversitärer Praxis der Ethnologie mit Rückgriff auf Bourdieus praxistheoretische Konzepte Habitus und Feld zu verstehen: Im Feld der universitären Ethnologie erwerben Ethnolog*innen einen Habitus (eine verkörperlichte Praxis), der oft nur mit Reibungen in außeruniversitäre Anwendungsfelder übertragen werden kann, in denen andere „praktische Logiken“ gelten. Hier werden von Ethnolog*innen zum Beispiel häufig Wissensformen und -praktiken erwartet, die in der universitären Praxis der Ethnologie kritisch reflektiert und hinterfragt werden. Dies soll insbesondere an einem Feld außeruniversitärer Praxis untersucht werden, das Ethnolog*innen erst seit kurzem offen steht: die Arbeit mit Flüchtlingen.
\end{abstract}

\section{Einleitung}

Praxis ist ein schillernder und vieldeutiger Begriff, der „in der Praxis“ ebenso eine zentrale Rolle spielt wie „in der Theorie“. Manchmal hat er eine wertende Konnotation, wenn etwa die Praxis der „bloßen Theorie“ gegenübergestellt wird. Die Gegenüberstellung von „Wissenschaft“ und „Praxis“ droht manchmal vergessen zu lassen, dass Wissenschaft selbst in erster Linie eine Praxis ist - selbst Naturwissenschaft, wie die Wissenschaftsforschung etwa Bruno Latours gezeigt hat. Verschiedene wissenschaftliche Disziplinen unterscheiden sich nicht nur durch ihre Gegenstände, sondern - vielleicht noch mehr durch ihre Praxisformen. So ist für die Ethnologie die Praxis der Feldforschung zentral. 
Für die Ethnologie ist aber Praxis auch ein ganz zentraler theoretischer Begriff. Die Theorieentwicklung der Ethnologie der letzten vier oder fünf Jahrzehnte lässt sich ohne einen Praxisbegriff, wie ihn etwa Sherry Ortner schon in den 1980er Jahren nachgezeichnet hat, nicht verstehen (Ortner 1984).

Was die „Theorie der Praxis“ - um mit Bourdieu (1979) zu sprechen - für das Verhältnis von universitärer und „praxisorientierter" Ethnologie bedeutet, ist das Thema dieses Beitrags. Ich versuche, die Differenz von universitärer und außeruniversitärer Praxis der Ethnologie mit Rückgriff auf Bourdieus praxistheoretische Konzepte Habitus und Feld zu verstehen. Kurz gesagt: Im Feld der universitären Ethnologie erwerben Studierende der Ethnologie einen Habitus (eine verkörperlichte Praxis), der oft nur mit Reibungen in außeruniversitäre Anwendungsfelder übertragen werden kann, in denen andere Habitusformen vorherrschen. Praxistheoretisch betrachtet ist es meines Erachtens zu kurz gegriffen, hier einfach nur wissenschaftliche Theorie und außerwissenschaftliche Praxis einander gegenüberzustellen. Tatsächlich sind die außerwissenschaftlichen Praxisformen mit spezifischen (theoretischen) Wissensformen verknüpft. Hier werden von Ethnolog*innen zum Beispiel häufig Wissensformen und -praktiken erwartet, die in der universitären Praxis der Ethnologie sehr kritisch reflektiert und hinterfragt werden. Ich werde dies an einem Feld außeruniversitärer Praxis diskutieren, das Ethnologinnen und Ethnologen zumindest in Bayern erst seit kurzem offensteht: dem Feld der Arbeit mit Geflüchteten.

\section{Theorie und Praxis}

Auch wenn die Unterscheidung „Wissenschaft“ und „Anwendung“ nicht einfach (in quasi-strukturalistischer Manier) äquivalent zur Unterscheidung „Theorie“ und „Praxis“ gesetzt werden kann, ist es hilfreich, von Bourdieus Konzeptualisierung von Theorie und Praxis auszugehen. Bourdieu entwickelt seinen Praxisbegriff als Kritik an einer theoretischen Vernunft, die in objektivistischer Weise ihre theoretischen Modelle als Grundlage für die außerwissenschaftliche und alltagsweltliche Praxis versteht. Dass diese Form des Objektivismus problematisch ist, weiß jeder, der einmal versucht hat, eine Sprache allein durch das Studium ihrer Grammatik sprechen zu lernen. Die Grammatik ist eine theoretische Objektivierung, die das Sprechen, die Kommunikation ebenso wenig leitet wie tatsächliche Heiratsbeziehungen auf der Basis von theoretischen Verwandtschaftsregeln geschlossen werden. In beiden Fällen ist die Praxis nicht einfach eine „Anwendung“ der Theorie, sondern ein eigenständiger Bereich, der nicht durch die theoretische Logik des Modells erklärt werden kann. Nach Bourdieu folgt die Logik der Praxis Erwägungen, die außerhalb des theoretischen Modells liegen - „praktischen“ Erwägungen eben. Jemand, der etwas sagt, will nicht die Grammatik der Linguisten befolgen, sondern einen Sinn ausdrücken und damit etwas erreichen. Genauso wollen Eltern, die die Ehen ihrer Kinder arrangieren, nicht ethnologische Modelle von Heiratsregeln umsetzen, sondern für 
ihre Familien möglichst „gute“ Beziehungen eingehen (siehe z. B. Bourdieu 1993:65-66). Theorie mit ihren Konzepten objektiviert und verdinglicht - sie essentialisiert (Bourdieu 1993: 71). Konzepte werden dabei mit der „Realität“ verwechselt (Bourdieu 1979: 159). Der sense pratique, der praktische Sinn (so der der Originaltitel von Bourdieus Werk „Sozialer Sinn“) kann all das missachten, was für die Theorie entscheidend ist: Logik, Widerspruchsfreiheit, Eindeutigkeit. Praxis ist gegenüber der Theorie autonom; sie lässt sich nie ganz theoretisch beschreiben oder anleiten. Praxis folgt nicht den Regeln der Theorie, sondern wird von den ihr eigenen Regeln, den Habitusformen strukturiert. Dies sind dauerhafte Dispositionen (Bourdieu 1993:98), die überwiegend unbewusst sind und den Erfordernissen des jeweiligen sozialen Feldes folgen. Sie sind damit ein „Produkt der Geschichte" (Bourdieu 1993: 101), ein Ergebnis der Erfahrung, die Bourdieu zufolge nicht notwendigerweise bewusst reflektiert wird. Der Habitus impliziert damit Wissensformen, die Michael Polanyis implizitem Wissen ähneln (Polanyi 1985), das weniger bewusst gelernt als durch ständige Wiederholung eingeübt wird. In diesem Sinn ist der Habitus inkorporiert, verkörperlicht - man kann nicht, oder nur sehr unbeholfen und vielleicht widerwillig, anders handeln. Bourdieu zufolge ist der Habitus verinnerlichte Struktur - er bildet gewissermaßen die strukturellen Bedingungen eines Feldes im individuellen Akteur ab, der damit die Struktur reproduziert, ohne dies als „Befolgen“ wahrzunehmen. Dieses Verhältnis von Habitus und Struktur verleiht dem Habitus in Bourdieus Konzeption eine gewisse Starrheit, weil er betont, dass der Habitus weitgehend unbewusst und unreflektiert bleibt. Dies muss jedoch nicht notwendigerweise so sein. Weiter unten werde ich postulieren, dass die Reflektion selbst zum Habitus werden kann.

In den Jahrzehnten seit Bourdieus Analyse hat sich das (ethnologische) Verständnis von Wissenschaft stark gewandelt. Ich möchte hier zwei Aspekte betonen: Erstens wissen wir nun, dass Wissenschaft selbst durch Habitusformen geprägt ist, und zwar nicht nur in dem Sinne, dass die Wissenschaft als Institution ein soziales Feld bildet, das im Hinblick auf Habitusformen untersucht werden kann, wie es etwa Bourdieu in Homo Academicus getan hat (Bourdieu 1988). Nein, auch die zentralen wissenschaftlichen Tätigkeiten der verschiedenen Disziplinen sind stark habituell geprägt. Latour hat das etwa für die Bodenkunde aufgezeigt (Latour 2002). Für die Ethnologie gilt dies besonders für die Feldforschung, die mindestens ebenso sehr impliziter Habitus wie explizite Methodologie ist. Dies wird besonders deutlich, wenn man Feldforschung unterrichtet (Sökefeld 2008). Der „Kern“ der Feldforschung kann nämlich nur sehr eingeschränkt formell, im Seminarraum, gelehrt werden. Es erfordert Übung im Feld, um einen sense pratique für die Feldforschung zu erlernen, der letztlich den produktiven Umgang mit unseren Interaktionspartnern in der Forschung ermöglicht - ganz wie bei anderen Formen sozialer Interaktion auch.

Zweitens hat die Ethnologie Bourdieus Kritik des Objektivismus - die sich in erster Linie gegen den Strukturalismus richtete - in der Kritik des Essentialismus weitgehend in ihre Theorie übernommen und seine Forderung, all die Theorien aufzugeben, „die explizit 
oder implizit die Praxis zu einer mechanischen, durch die vorhergehenden Bedingungen unmittelbar determinierten Reaktionsformen stempeln“ (Bourdieu 1979:169) in vieler Hinsicht umgesetzt. Verwandtschaftsethnologie erschöpft sich zum Beispiel nicht mehr in der Ausarbeitung von Regeln und Modellen, die davon ausgehen, dass ihnen die konkreten Verwandtschaftsbeziehungen folgen, sondern stellt die Frage, wie und in welchem Kontext Beziehungen und Verbundenheit, relatedness (Carsten 2000), hergestellt werden. Für die Politikethnologie ist schon seit den 1950er Jahren die Untersuchung von politischen Prozessen, d. h. von praktischem politischen Handeln, wichtiger als die zuvor dominante Definition und Analyse von Strukturen und Typologien (Kurtz 2001, Kap. 7). Gleiches gilt für Konzepte von Kultur oder Identität. Konstruktivistische Konzepte von Identität etwa haben längst die praktische Unschärfe und Vieldeutigkeit von Identitätszuschreibungen und Ethnonymen in die Theorie inkorporiert (Finke/Sökefeld, 2018). Eine Theorie der Ethnizität zum Beispiel, der es vor allem darum ginge, ein widerspruchsfreies Modell eindeutig abgegrenzter Ethnonyme zu entwickeln, würde wohl heute von keiner ethnologischen Fachzeitschrift zur Publikation angenommen.

In der Ethnologie hat sich ein anti-essentialistischer Habitus entwickelt. Dies ist sehr gut an Studierenden im Bachelor zu beobachten, die anfangs unbekümmert essentialistische Zuschreibungen verwenden, im Laufe des Studiums jedoch die anti-essentialistische Kritik übernehmen, und zwar nicht nur intellektuell, sondern durchaus auch „verkörperlicht": Sie können einfach nicht mehr in essentialistischen Begriffen reden - auch außerhalb der Universität, etwa in ihren Familien oder Freundeskreisen. Der Anti-Essentialismus ist tatsächlich eine „einverleibte Objektivität“ (Bourdieu 1979:170), denn im Feld der universitären Ethnologie ist Essentialismus objektiv unhaltbar: Wer etwa in Hausarbeiten essentialistische Positionen vertritt, wird per schlechter Bewertung abgestraft. Die Konfrontation mit Essentialismen außerhalb dieses Feldes verursacht dann fast eine Art körperliches Unwohlsein. Allerdings ist dieser Habitus eben nicht vor-reflexiv und unbewusst, sondern eher ein inkorporierter „Drang zur Reflexion“.

\section{Ethnologie und ihre Anwendungsfelder}

Wie können wir vor diesem Hintergrund das Verhältnis der Ethnologie zu ihren Anwendungsfeldern denken? Gemäß Bourdieus Kritik des Objektivismus ist dieses Verhältnis nicht eins, in dem (ethnologische) Theorie auf ein „Praxisfeld“ angewendet wird; denn die Praxis ist keine Umsetzung oder Anwendung der Theorie, sondern folgt ihren eigenen Logiken, die durch Habitusformen strukturiert sind. Darüber hinaus ist Ethnologie eben selbst nicht nur Theorie, sondern auch Praxis. Und genauso erschöpfen sich die Anwendungsfelder keineswegs nur in Praktiken, sondern sind auch durch eigene Formen und Annahmen von Wissensproduktion bestimmt, also auch durch Theorie. Bourdieu betont, dass Habitusformen in spezifischen Feldern entstehen und auch nur dort „passen“, dass also ein dem Habitus entsprechendes Handeln von Interaktionspartnern 
nur innerhalb des spezifischen Feldes problemlos verstanden und passend erwidert wird (Bourdieu 1993: 107-108). Außerhalb des Feldes der Ethnologie eckt Handeln, das dem ethnologischen Habitus folgt, plötzlich an - die nicht-ethnologischen Habitus-Formen eines Anwendungsfeldes und die damit verbundenen Erwartungen produzieren Friktionen.

Diese Reibungen sind auch bei einem Anwendungsfeld deutlich, das auf den ersten Blick besonders geeignet für die Anwendung ethnologischen Wissens erscheint: bei der Interkulturellen Kommunikation. Kulturelle Unterschiede aufzuzeigen, sie zu deuten und damit verstehbar zu machen, ist ein zentrales Anliegen der akademischen Ethnologie. Dennoch lässt sich ethnologisches Wissen nicht einfach und ungebrochen ins Praxisfeld der Interkulturellen Kommunikation übertragen. Man kann zwei Kategorien ethnologischen Wissens über Kultur unterscheiden: Kulturwissen und Metawissen (Sökefeld 2009). Kulturwissen meint Wissen über kulturelle Vorstellungen, Praktiken, Diskurse, Institutionen etc. Kulturwissen hat eine gewisse Tendenz zur Essentialisierung, vor allem dadurch, dass seine Elemente gerne gewissen Kollektiven - ethnischen Gruppen, Nationen - zugeschrieben werden. Metawissen bezieht sich dagegen auf Wissen über Prozesse der sozialen Produktion von Kultur und kultureller Differenz, über den (identitäts-)politischen Gebrauch von Kultur, über Dynamiken von othering, das Verhältnis von Kultur und Macht usw. Kulturelles Metawissen resultiert aus der Analyse der Produktion von Differenz in einer Welt miteinander verknüpfter und interdependenter kultureller, sozialer, wirtschaftlicher und politischer Räume, die Akhil Gupta und James Ferguson vor zwanzig Jahren anmahnten, um die gängige und scheinbar so selbstevidente Zuschreibung von Differenz zu als räumlich voneinander getrennt vorgestellten „Kulturen“ aufzubrechen (Gupta und Ferguson 1997:43). Obwohl beides letztlich nicht voneinander zu trennen ist, ist das Metawissen für die Ethnologie fundamentaler, da Metawissen über die Prozesse der Produktion von Kultur die Grundlage für die Einordnung und Gültigkeit des Kulturwissens ist (Sökefeld 2009:27). Der anti-essentialistische ethnologische Habitus ist vor allem mit dem Metawissen über Kultur verknüpft - das Metawissen bricht den potentiellen Essentialismus des Kulturwissens auf.

Was aber im Praxisfeld interkultureller Trainings von Ethnologinnen und Ethnologen in erster Linie erwartet wird, ist die Vermittlung von Kulturwissen. Gibt man einem Japaner zur Begrüßung die Hand? Wie sehen die Verhandlungsstrategien chinesischer Geschäftspartner aus? Sind französische Unternehmen eher hierarchieorientiert? Schon der „methodologische Nationalkulturalismus“ solcher Fragen stößt dem anti-essentialistischen Habitus der Ethnologie massiv auf. Wer aber als (ethnologischer) interkultreller Trainer versucht, erst einmal die Idee einer japanischen Nationalkultur zu dekonstruieren, kommt bei den Teilnehmer*innen des Trainings überwiegend nicht gut an. Ethnologische Trainer*innen können versuchen, sich dahin zu retten, zunächst einmal die eigenen kulturellen Erwartungen und Konstruktionen zu thematisieren. Letztlich werden sie sich aber damit auseinandersetzen müssen, dass die Teilnehmer*innen und Auftraggeber*innen interkultureller Trainings die Vermittlung klarer Regeln und Handlungs- 
anweisungen in Bezug auf „andere Kulturen“ erwarten. Sie erwarten eine Art „Grammatik“ des Handelns, obwohl der sozial kompetente Umgang mit kulturellen Unterschieden ebenfalls eher einen sens pratique erfordert, den man aber nicht einfach in einem Seminar lehren kann.

\section{Ethnolog*innen in der Sozialen Arbeit mit Flüchtlingen - Reflektionen und Erfahrungen aus Bayern}

Bis zum Frühjahr 2015 waren Ethnolog*innen in Bayern von Stellen in der Asylsozialberatung und anderen Feldern der Arbeit mit Flüchtlingen bei Trägern der Freien Wohlfahrtspflege - vom Freistaat Bayern finanziert - ausgeschlossen. Sie verfügten nicht über das Qualifikationsprofil, das für diese Stellen von den Trägern genauso wie vom Bayerischen Sozialministerium vorausgesetzt wurde, nämlich Abschlüsse in Sozialpädagogik, Sozialarbeit oder Pädagogik. Anfang 2015 waren jedoch auf dem Arbeitsmarkt aufgrund des schnellen Wachsens der Flüchtlingszahlen und des steigenden Bedarfs kaum noch Menschen mit entsprechender Qualifikation, die im Asylbereich arbeiten wollten, verfügbar. Eine Absolventin des Instituts für Ethnologie der LMU - ich nenne sie Anna ${ }^{1}-$ bewarb sich Anfang 2015 auf eine Stelle in der Asylsozialberatung bei einem konfessionellen Träger im Umland von München. Der Träger wollte Anna einstellen, bekam dafür jedoch keine Genehmigung, da sie eben keine (Sozial-)Pädagogin war. Andere geeignete Bewerber*innen gab es jedoch nicht. Anna fand heraus, dass das Bayerische Sozialministerium die Einstellung aufgrund ihrer „falschen“ Qualifikation blockierte. Daraufhin schrieb ich an die entsprechende Stelle im Ministerium einen Brief, in dem ich für den Einsatz von Ethnologinnen und Ethnologen in der Arbeit mit Flüchtlingen warb und ihre Qualifikationen erklärte. Auf diesen Brief bekam ich nie eine Antwort, allerdings gab das Ministerium den Widerstand gegen Annas Einstellung auf, wohl auch, weil inzwischen klargeworden war, dass der Bedarf der Asylberatung und der Betreuung von Flüchtlingen nur mit ausgebildeten Sozialpädagog*innen ${ }^{2}$ schon damals nicht mehr zu decken war. Später erfuhr ich, dass mein Brief an die Landesarbeitsgemeinschaft der Freien Wohlfahrtspflege Bayern zur Stellungnahme weitergeleitet worden war. In ihrer Antwort an das Ministerium, die mir vorliegt, betont die Landesarbeitsgemeinschaft, dass nur Menschen mit einem sozialpädagogischen Abschluss in vollem Umfang über die für eine Tätigkeit in der Asylsozialberatung erforderlichen Qualifikationen verfügten. Dabei wurde besonders auf Kenntnisse des Rechts, aber auch auf Techniken der Gesprächsführung verwiesen. Angesichts der Situation auf dem Arbeitsmarkt war die Landesarbeitsgemeinschaft jedoch bereit, Ausnahmegenehmigungen für Menschen ohne die klassischen Ab-

1 Alle Namen sind Pseudonyme.

2 Der Einfachheit halber fasse ich im Weiteren in dieser Kategorie alle entsprechenden Qualifikationen (auch Sozialarbeiter*innen und Pädagog*innen) zusammen. 
schlüsse zuzulassen - auch für Ethnolog*innen, sofern sie an Fortbildungen teilnehmen. Ethnolog*innen wurden also nur als „zweite Wahl“ betrachtet und später auch tatsächlich oft so behandelt: Sie wurden tariflich schlechter eingruppiert, nur befristet beschäftigt, und wenn Stellen zur Verlängerung oder Entfristung anstanden, wurden meist Sozialpädagog*innen bevorzugt. Aber die Flüchtlingszahlen stiegen weiter und so wurden immer mehr Ethnolog*innen in diesem Arbeitsfeld eingestellt. Inzwischen haben nach meiner Kenntnis etwa zwanzig Absolvent*innen des Münchner Instituts - mit Bachelor-, Master- und Magisterabschluss Ethnologie - Stellen in verschiedenen Bereichen der Beratung und Betreuung von Flüchtlingen gefunden, sei es in der direkten Asylsozialberatung, in der Betreuung in Unterkünften, in der Freizeitarbeit mit Kindern und Jugendlichen, in der Bildungsförderung oder in der Koordination von Ehrenamtlichen. Seit Herbst 2015 gibt es am Münchner Institut einen regelmäßigen Erfahrungsaustausch über die Arbeit in diesen Tätigkeitsfeldern. Auf dieser Basis und auf der Grundlage einiger weiterer Gespräche mit Ethnologinnen in der Flüchtlingsarbeit möchte ich im Folgenden über die Arbeit von Ethnologinnen in diesem Bereich reflektieren. ${ }^{3}$

Bei den ersten Gesprächsrunden des Erfahrungsaustauschs fiel auf, dass die Arbeit im sozialpädagogischen Kontext ständig thematisiert wurde. Diskussionswürdig war den Ethnologinnen ${ }^{4}$ weniger der Umgang mit den Geflüchteten als die Interaktion mit Sozialpädagog*innen. Sie erschienen den Ethnologinnen weit "fremder" als Menschen aus Syrien, Eritrea oder Afghanistan. Besser gesagt, gab es gegenüber den Sozialpädagog*innen vielleicht eine andere Art von Fremdheit, auf die die Ethnologinnen viel weniger vorbereitet waren als auf die klassische „kulturelle Differenz" zu Menschen aus anderen Herkunftsländern, mit der sie sich im Studium auseinandergesetzt haben. Problematisch erschien ihnen vor allem der in ihrer Wahrnehmung vergleichsweise unreflektierte Umgang mit zentralen Konzepten, zum Beispiel mit „Integration“ und natürlich auch mit „Kultur“. Hier war die Kernkompetenz der Ethnologie und damit auch der ethnologische Habitus berührt. Im sozialpädagogischen Kontext war die „Integration“ von Geflüchteten eine „normale“ Forderung, ein (politisch) vorgegebenes Ziel, das man mit bestimmten Maßnahmen versuchte zu erreichen, das an sich aber kaum thematisiert und auch nicht hinterfragt wurde. Das aber stieß den Ethnologinnen auf: Für sie war „Integration" problematisch, weil der Begriff in Beziehungen von Ungleichheit eingeschrieben ist und Machtverhältnisse zementiert. Im Rahmen des Integrationsparadigmas der deutschen Migrationspolitik ist klar, wer sich integrieren muss. Integration ist eine Forderung, die an „die anderen“, an Flüchtlinge und Migranten, gerichtet ist. Noch dazu ist „Integration“ ein normativer Begriff, der Migranten in „gute“ (= „Integrationswillige“) und „schlechte“ (= „Integrationsverweigerer“) einteilte. Obwohl im politischen Diskurs

3 Ich danke den Teilnehmer ${ }^{\star}$ innen der Gesprächsrunden ganz herzlich für ihre Erfahrungsberichte - insbesondere den drei Ethnologinnen, die ich auch noch separat interviewen konnte.

4 Bis auf eine Ausnahme handelt es sich tatsächlich um Frauen. 
durchaus hin und wieder betont wird, dass Integration ein „beiderseitiger“ Prozess und daher nicht mit Assimilation gleichzusetzen sei, bleiben Integrationsforderungen und Integrationspolitik in der Praxis einseitig an „die anderen“ gerichtet; im Kern wird von Migranten letztlich doch Assimilation erwartet. Noch dazu reproduziert die an Migranten gerichtete Integrationsforderung ständig die Grenze zwischen „uns“ (den Deutschen) und „den anderen“ (den Migranten) und verhindert durch diese ständige Ausgrenzung letztlich das Ziel der „Integration“ (Sökefeld 2007).

Das Integrationsparadigma einfach zu akzeptieren und in ihren Einrichtungen entsprechend zu handeln, war für die Ethnologinnen schwierig. Meine Gesprächspartnerinnen betonten mehrfach, dass es nicht darum ging, zu behaupten, dass alle Sozialpädagog*innen „Integration“ unhinterfragt akzeptierten, aber dass doch deutlich wurde, dass die skeptische Reflektion nicht unmittelbar zum Umgang mit dem Begriff dazu gehörte. Ähnlich war der Umgang mit dem Begriff „Kultur“. Sozialpädagog*innen hatten kaum genaue Vorstellungen über Ethnologie, wussten aber schon, dass Ethnologie mit „Kultur“ zu tun hat. Und so wurden die Ethnologinnen als „Expertinnen für Kultur“ betrachtet, wobei Kultur eindeutig im Sinn (nationaler) Herkunftskultur verstanden wurde. Von Ethnolog*innen wurde erwartet, kulturelle Erklärungen für alle möglichen Probleme zu liefern - also Kulturwissen. Als sich zum Beispiel in einer Unterkunft mehrere afghanische Jugendliche ritzten, wurde die Frage gestellt: Warum ritzen sich afghanische Jugendliche? Kann man das mit afghanischer Kultur erklären? Die Ethnologin erklärte zuerst, dass man angesichts der kulturellen Diversität Afghanistans kaum von einer „afghanischen" Kultur sprechen kann und gab dann den Rat, mit den Jugendlichen selbst darüber zu sprechen. Sie vermutete, dass einer mit dem Ritzen angefangen hatte und die anderen es nachmachten, weil sie alle in einer sehr belasteten Situation steckten. Der Aufenthalt der Jugendlichen war nicht gesichert, möglicherweise stand ihre Abschiebung bevor. Die Ethnologin gab also auf eine „theoretische“, objektivierende Frage interessanterweise eine „praktische“ Antwort: Redet mit den Jugendlichen!

Eng verbunden mit der Erwartung kultureller Erklärungen ist eine gewisse Fixierung auf die Herkunft von Menschen. Manche Einrichtungen führen Statistiken, die die Herkunft ihrer „Klienten“ festhalten. Oft werden „Kultur“ und nationale „Herkunft“ - afghanische Kultur und Afghanistan - gleichgesetzt und auch nicht selten mit körperlichen Merkmalen assoziiert. Nach Augenschein wird entschieden, wer „fremd“ ist und wer nicht. Dies gilt nicht nur in Bezug auf Geflüchtete. Die Ethnologin Martha erzählte von den Erfahrungen einer Sozialpädagogin, deren Eltern nach Deutschland eingewandert waren, und deren Aussehen nicht dem „herkunftsdeutschen“ Stereotyp entsprach. Bei Netzwerktreffen und ähnlichen Gelegenheiten wurde sie von anderen Sozialpädagog*innen, die sie noch nicht kannten, in der Regel nicht als Kollegin, sondern als Klientin identifiziert, die, wenn sich herausstellte, dass sie doch Sozialpädagogin war, sofort nach ihrer „Herkunft“ gefragt wurde. Dies ist natürliche eine gängige Ausgrenzungserfahrung von „Menschen mit Migrationshintergrund“ (auch dies ein tendenziell ausgrenzender 
Begriff) in Deutschland, die keineswegs auf das „sozialpädagogische Milieu“ beschränkt ist. Das Beispiel zeigt aber, dass in diesem Milieu die gängigen Zuschreibungen und Normalitätserwartungen auch nicht besonders hinterfragt und aufgebrochen werden.

Insgesamt war die Wahrnehmung der Ethnologinnen, dass Soziale Arbeit mit eher starren Kategorien operiert. Ein Beispiel dafür waren auch die „Hilfeplangespräche“, die mit minderjährigen Geflüchteten geführt werden müssen. Hilfeplangespräche sind ein gesetzlich vorgeschriebenes Verfahren der Kinder- und Jugendhilfe, bei dem Entwicklungsziele für die betroffenen Jugendlichen individuell festgelegt und anhand eines Fragenkatalogs regelmäßig überprüft und beurteilt werden. Dabei wird ein Fragenkatalog verwendet, der nicht immer spezifisch an die Situation minderjähriger Flüchtlinge angepasst ist, der nach Meinung der Ethnologinnen häufig nicht passt und auch für die betroffenen Jugendlichen selbst nicht immer einen Sinn ergibt. Nach Einschätzung der Ethnologinnen können sie die gesetzten Ziele in vielen Fällen gar nicht erreichen, obwohl die Beurteilung für die Jugendlichen essentiell ist, da z. B. die Art ihrer Unterbringung und der Grad ihrer Betreuung davon abhängig gemacht werden können.

An den Hilfeplangesprächen wird auch eine weitere Problematik deutlich: Die Sozialpädagog*innen wissen immer schon, „wo es hingehen soll“, wie das eine der Ethnologinnen - ich nenne sie Maria - ausdrückte. Die Schwierigkeit besteht dabei für sie nicht in der Handlungsperspektive der Sozialen Arbeit an sich, sondern in der Normativität, die in diese Handlungsperspektive immer schon eingeschrieben ist und die sich mit der für die Ethnologie doch immer noch eher relativistischen Haltung reibt. Man weiß, wo es hingehen soll und was z. B. für die betroffenen Jugendlichen gut ist. In der Wahrnehmung der Ethnologinnen orientiert sich dieses Wissen aber weniger an den Wünschen und Zielen der Jugendlichen - und wird auch nicht in Auseinandersetzung mit ihnen erarbeitet - als an den vorgegebenen Zielen der Sozialen Arbeit - wie zum Beispiel dem Ziel der „Integration“. Es geht darum, die Jugendlichen „passend zu machen“, sagte eine meiner Gesprächspartnerinnen. Mit Michel Foucault (1994) gesprochen geht es hier um Disziplinarpraktiken, die im sozialpädagogischen Kontext oft nicht hinterfragt, sondern einfach umgesetzt werden.

Diese Beispiele machen deutlich, dass es hier nicht um einen Gegensatz von Theorie (=Ethnologie) und Praxis (=Soziale Arbeit) geht, sondern um verschiedene Ausrichtungen der Praxis, bzw. um verschiedene Habitusformen, die die Praxis leiten und die mit verschiedenen Wissenspraktiken verknüpft sind. Obwohl die Ethnolog*innen als Expert*innen für das Kollektive („Kultur“) gelten, betonen sie interessanterweise in der Regel die Notwendigkeit des genauen Blicks auf das Individuum und seinen Kontext, die Bedeutung der biographischen Perspektive ${ }^{5}$, und stellen die Kollektivierungen der Sozial-

5 Man kann hier durchaus Anklänge an Lila Abu-Lughods „ethnography of the particular“ sehen, die sie in ihrem vielgelesenen Artikel Writing Against Culture als Strategie gegen Essentialisierungen vorschlägt (AbuLughod 1991). 
pädagog*innen - sei es in der Form von Herkunftszuschreibungen oder normativen Handlungsperspektiven - in Frage. Anstatt kritische Situationen etwa per Anrufung von Kulturwissen zu erklären, fordern sie gewissermaßen auf, eine kleine Feldforschung zu machen: Geht hin, schaut hin, fragt nach!

Das Feld der Sozialen Arbeit mit Flüchtlingen ist jedoch nicht einheitlich. Die hier genannten Erfahrungen beziehen sich in erster Linie auf das Sub-Feld der Betreuung von Geflüchteten, sei es in Unterkünften oder Freizeiteinrichtungen, und zwar besonders von Minderjährigen oder jungen Erwachsenen. Das Handeln der Betreuer*innen ist hier stark durch gesetzliche und administrative Vorgaben eingeschränkt und die Normativität wie das Ziel der „Integration“ - ist in diese Vorgaben eingeschrieben. Die sozialpädagogische Handlungsperspektive der Betreuung findet innerhalb des vorgegebenen Systems statt. Sie ist affirmativ; die kritische Reflexion dieses Rahmens wäre schon subversiv. Und diese Subversion, die viel von dem in Frage stellen würde, was sie während ihres Studiums gelernt haben, liegt vielen Sozialpädagog*innen offensichtlich weniger nahe als den Ethnolog*innen, die im Studium immer wieder mit der kritische Analyse von Machtverhältnissen zu tun haben.

Die Ethnologin Hanna, die im Sub-Feld der Asylsozialberatung arbeitet, hat dagegen andere Erfahrungen in ihrem Team gemacht. Asylsozialberatung findet zumindest teilweise auch gegen das System statt, denn es geht darum, für die Asylbewerber angesichts der massiven rechtlichen und administrativen Einschränkungen möglichst viele Ressourcen und Optionen herauszuholen. ${ }^{6}$ Hanna erzählte, dass sie vor Beginn ihrer Tätigkeit in der Beratungsstelle die Befürchtung hatte, im sozialpädagogischen Umfeld dort mit stark „erzieherischen“ Ansätzen und Perspektiven konfrontiert zu werden. Diese Befürchtung bewahrheitete sich jedoch nicht: Die Berater*innen - gleich welche Ausbildung sie mitbrachten - waren ganz überwiegend darauf aus, die Autonomie der Asylsuchenden zu unterstützen und ihnen Handlungsmöglichkeiten trotz aller Einschränkungen zu eröffnen. Starre Konzepte, Kulturwissen oder Normativität spielen hier kaum eine Rolle. Stattdessen geht es darum, im sich ständig ändernden Gewirr rechtlicher und administrativer Regeln und Prozeduren Strategien für das Handeln zu entwickeln, also - in Bourdieus Sinn - um die Logik der Praxis.

Auf meine Frage, welche der Fähigkeiten und Kenntnisse, die sie im Ethnologiestudium erworben hat, für ihre Beratungstätigkeit am wichtigsten ist, nannte Hanna die Fähigkeit zum Perspektivwechsel: das Bemühen, sich in die Position der anderen - in diesem Fall der Flüchtlinge - hineinzuversetzen, im Versuch, ihre Situation, ihre Erfahrungen, aber auch ihre Ziele und Ambitionen zu verstehen und zu respektieren. Sie betonte, dass ihre sozialpädagogischen Kolleg*innen diese Haltung teilten, die vom Feld der Beratungs-

6 Der subversive Charakter der Asylsozialberatung wurde offensichtlich, als im März 2017 das Bayerische Sozialministerium Trägern der Beratung damit drohte, die finanzielle Förderung einzustellen, sollten sie Asylbewerbern auch darin beraten, wie Abschiebungen verhindert werden können. Siehe dazu Süddeutsche Zeitung 2017 und Münchner Merkur 2017. 
arbeit gefordert wird, in dem es tatsächlich kaum festgelegte Handlungsanweisungen gibt, die einfach „in die Praxis“ umgesetzt werden könnten. Dies ist im Feld der Betreuungsarbeit jedoch anders. Hier geht es weniger darum, sich in die Perspektive der geflüchteten Jugendlichen hineinzuversetzen, als die Geflüchteten in das System der Jugendarbeit zu versetzen. Die Vorgaben sind klar und müssen umgesetzt werden, sonst fallen die Jugendlichen aus dem System heraus. Der ethnologische Habitus der Reflexion, des Hinterfragens und des Perspektivwechsels wirft in diesem Feld eher Probleme auf. ${ }^{7}$

\section{Schluss}

Die Erfahrungen von Ethnolog*innen in der Arbeit mit Flüchtlingen machen deutlich, dass das Verhältnis von Ethnologie und ihrer Anwendung komplexer ist, als landläufig gedacht. Es geht nicht einfach um die Anwendung von (ethnologischer) "Theorie“ in der „Praxis“, sondern um das Zusammentreffen von zwei (oder mehr) Praxisformen mit ihrem jeweiligen Habitus in spezifischen Berufsfeldern. Der Habitus, den Ethnologinnen und Ethnologen durch ihr Studium erwerben, ist heute eindeutig von anti-essenzialistischer Reflexion geprägt. Anti-Essenzialismus ist nicht nur eine theoretische Perspektive, sondern mindestens ebenso sehr eine Wissenspraxis, die zur ständigen Reflexion - einer Tätigkeit - aufruft.

Dies war vermutlich nicht immer so: Der Habitus kann sich verändern. Eine meiner Gesprächspartner*innen arbeitet auch mit einer Ethnologin zusammen, die ihr Studium gut zwanzig Jahre früher abgeschlossen hat und häufig kollektive, essentialisierende Zuschreibungen verwendet, die damals in der Ethnologie als weit weniger problematisch galten. Damals herrschte als Habitus vermutlich noch die Objektivierung vor, die Bourdieu anprangert. ${ }^{8}$ Außerhalb der Ethnologie ist das Bild der Ethnologie als Wissenschaft der Kulturen immer noch dominant - wie die Erwartungen, die an Ethnolog*innen auch im Feld der Arbeit mit Flüchtlingen gerichtet werden, zeigen. Diese Erwartungen reproduzieren allgemeine gesellschaftliche Wissensformen und diskursive Praktiken, die zum Beispiel Menschen nach „Herkunft“ kategorisieren und ihnen auf dieser Basis bestimmte Eigenschaften und Handlungsweisen zuschreiben.

Ich denke, die komplexere Analyse des Verhältnisses der Ethnologie zu ihren Anwendungsfeldern, die das Verhältnis nicht zu einem von „Theorie“ zu „Praxis“ reduziert, ist in mehrfacher Hinsicht sinnvoll. Zum einen verhindert sie die Entwertung der Ethnologie

7 Interessanterweise äußerten einige der Ethnologinnen im Feld der Betreuung den Verdacht, dass geflüchtete Jugendliche sich manchmal nur strategisch zu den Vorgaben der Jugendarbeit verhalten, sich die implizierte Normativität aber nicht tatsächlich zu eigen machen.

8 Ich bin mir bewusst, dass ich mit diesem Satz den Objektivismus - anders als Bourdieu - vom Bereich der Theorie in die Praxis ausdehne. Tatsächlich folgen aus dem theoretischen Ziel des Objektivismus bestimmte Wissenschaftspraktiken, wie zum Beispiel die schematischen Darstellungsweisen, die Bourdieu erwähnt und kritisiert. 
aus der Perspektive des Anwendungsfelds als „bloße Theorie“, die im Anwendungsfeld „der Praxis“ untergeordnet werden muss. ${ }^{9}$ Zum anderen ermöglicht uns das Aufgaben der simplifizierenden Vorstellung, Ethnologie sei Theorie, die in der Praxis angewendet werden kann (oder muss), eine bessere, realistischere Vorbereitung der Studierenden auf die verschiedenen Anwendungsbereiche, die zu erwartende Schwierigkeiten in verschiedenen Anwendungsfeldern - nicht nur in der Arbeit mit Flüchtlingen - klarer benennen kann. Letztlich kann daraus, hoffentlich, ein größeres Selbstbewusstsein von Ethnologinnen und Ethnologen resultieren, dass angesichts der „Zwänge der Praxis“ ethnologische Perspektiven nicht überwiegend über Bord geworfen werden müssen, sondern dass sie tatsächlich wichtige Beiträge zu Anwendungsfeldern leisten können.

\section{Literatur}

Abu-Lughod, Lila (1991): Writing against Culture. In: Fox, Richard G. (Hg.): Recapturing Anthropology. Working in the Present. Santa Fe: School of American Research Press, S. 137-162.

Bourdieu, Pierre (1979): Entwurf einer Theorie der Praxis. Frankfurt: Suhrkamp.

Bourdieu, Pierre (1988): Homo Academicus. Frankfurt: Suhrkamp.

Bourdieu, Pierre (1993): Sozialer Sinn. Kritik der theoretischen Vernunft. Frankfurt: Suhrkamp.

CARsten, Janet (Hg.) (2000): Cultures of Relatedness. New Approaches to the Study of Kinship. Cambridge: Cambridge University Press.

FINKE, Peter / Sökefeld, Martin (2018): Identity in Anthropology. In: Callan, Hillary (Hg.): The International Encyclopedia of Anthropology. Wiley \& Sons. https://doi.org/10.1002/9781118924396.wbiea 2142.

GuptA, Akhil / Ferguson, James (1997): Beyond 'Culture'. Space, Identity, and the Politics of Difference. In: Gupta, Akhil/ Ferguson, James (Hg.): Culture Power Place. Explorations in Critical Anthropology. Durham: Duke University Press, S. 33-51.

Kurtz, Donald V. (2001): Political Anthropology. Paradigms and Power. Boulder: Westview Press.

LAtour, Bruno (2002): Die Hoffnung der Pandora. Frankfurt: Suhrkamp.

MÜNCHNER Merkur (2017): Tipps gegen Abschiebung. Ministerium droht Flüchtlingshelfern. 17.05.2017: https://www.merkur.de/lokales/muenchen/stadt-muenchen/fluechtlinge-verbaende-wehren-sich-gegeneinschuechterung-durch-staatsregierung-8315686.html [Zugriff am 27.10.2017].

Ortner, Sherry B. (1984): Theory in Anthropology since the Sixties. In: Comparative Studies in Society and History, 26, S. 126- 166.

POLANYI, Michael. 1985. Implizites Wissen. Frankfurt: Suhrkamp.

SöKefeld, Martin (2007): Zum Paradigma kultureller Differenz. In: Johler, Reinhard / Thiel, Ansgar/Schmid, Josef/Treptow, Rainer (Hg.): Europa und seine Fremden. Die Gestaltung kultureller Vielfalt als Herausforderung. Bielefeld: transcript Verlag, S. 41-57.

Sökefeld, Martin (2008): Zur Theorie und Praxis der Feldforschungsausbildung in der Ethnologie. In: Ethnoscripts, 10 (2), S. $114-133$.

SöKefelD, Martin (2009): Ethnologie und interkulturelle Kommunikation. In: Koch, Gertraud / Franke, Amelie (Hg.): Kulturelle Vielfalt als Gestaltungsaufgabe. St. Ingbert: Röhrig Universitätsverlag, S. 23-31.

SÜDDEUTSCHE ZEITUNG (2017): Empörung über Sozialministerium. Wohlfahrtsverbände warnen vor Eingriff in Flüchtlingsberatung. 8.3.2017: http://www.sueddeutsche.de/bayern/asylpolitik-empoerung-ueber-sozialministerium-1.3410753 [Zugriff am 27.10.2017].

9 Sie schärft letztlich auch den Blick auf Machtpraktiken, mit denen die in einem Feld etablierten Disziplinen Ethnolog*innen zum Beispiel mit Verweis auf mangelnde oder "falsche“ Qualifikationen auszugrenzen versuchen. 